\title{
Gingival Absorption of Rat Periodontal Disease Model and Rat Tooth Movement Model
}

Y. TAI

Department of Stomatology, The Second Hospital of Tianjin Medical University, Tianjin, 300211, China

Tai et al.: Gingival Absorption of Rat Periodontal Disease Model and Rat Tooth Movement Model

In order to study the relationship between periodontal disease and alveolar bone resorption and the relationship between orthodontic treatment and alveolar bone resorption, two models of periodontal disease rats and tooth movement rats were constructed. The clinical diagnosis of gingival index, plaque index and depth of probing in the rat model of periodontal disease confirmed the successful construction of the periodontal disease model, and then the hematoxylin and eosin staining of the maxillary first molar tissue section revealed periodontal disease can cause aggregation of cementoclast and observation of nonstained sections showed that the area of absorption of periodontal disease rats was significantly increased compared with healthy rats. In this study, the rat model of tooth movement was also used. The left side of the maxillary teeth of the rat was injected with Ibanonate sodium phosphate in the experimental group, and the right side was injected with normal saline as the control group. It was found that in the tissue sections treated with Ibanonate sodium phosphate, the cementoclast were reduced and the degree of fang alveolar bone resorption was reduced. It indicated that the treatment has an inhibitory effect on fang alveolar bone resorption, which proved that Ibanonate sodium phosphate can be used in the treatment of orthodontic treatment of root resorption.

Key words: Periodontal Disease, orthodontic treatment, cementoclast, cementum resorption

Alveolar bone resorption is similar to bone resorption ${ }^{[1]}$, the only difference is that bone resorption is mediated by osteoclasts, and cementum resorption is mediated by cementoclast, and the cell morphology and working principle of the two are similar ${ }^{[2]}$. The diameter of the cementoclast ranges from $30 \mathrm{~nm}$ to $100 \mathrm{~nm}$. There are multiple nuclei, generally round nuclei, and the nuclear membrane is flat. The nucleolus is generally one or two. The cytoplasm of cementoclast is basophilic when they are naive, and the cytoplasm is eosinophilic after maturity. With the aging of cementoclast, the cytoplasmic acidophily is getting stronger and stronger, mainly because of its abundant lysosomes, mitochondria and ribosomes in the cytoplasm ${ }^{[3]}$. Usually the cementum is protected by extracellular matrix (ECM) such as the gingiva, and the cementum cells can't be in contact with the root bone or alveolar bone. However, if these protective tissues are destructed by periodontal disease or orthodontic treatment, the osteoclasts adhere to the surface of the root or alveolar bone, and then the polarized cells are in intimate contact with the bone matrix and cause a specific microenvironment to mediate the bone resorption. When the bone resorption lacuna is formed, the cementoclast will shift to another position and continue to absorb or die on the next bone absorbing surface. There are many causes of alveolar bone resorption ${ }^{[4]}$, mainly including periodontal disease, orthodontic treatment, etc.

Periodontal disease is a chronic inflammatory disease caused by external environmental factors such as bacteria, trauma, oral hygiene, and maxillofacial deformity, which can result in loss of grounded tissue attachment, alveolar bone erosion and even tooth $\operatorname{loss}^{[5]}$. The main pathological feature of periodontal disease is the destruction of the gingiva and periodontal support tissue - the extracellular matrix ${ }^{[6]}$. ECM contains a variety of collagen fibers, and the gingiva and these collagen fibers will tightly wrap the roots to protect the roots from external pathogens or the external environment, ensuring healthy teeth. When suffering from periodontal disease, the ECM is destroyed, causing the periodontal connective tissue such as the gingiva to be destroyed, exposing the alveolar bone and the root 
bone, and the alveolar bone resorption phenomenon occurs $^{[7]}$.

Malocclusion is a kind of oral disease caused by genetic and environmental factors. The patient's maxillofacial development is imperfect, which has different effects on the appearance of the patient, the health and function of the oral cavity, and may even cause mental illness ${ }^{[8]}$. With the development of social economy and people's pursuit of quality of life, oral orthodontic treatment has increasingly entered people's lives. After treatment, the oral beauty and function have been improved, but the complications of orthodontics have followed. Alveolar bone resorption is the main complication; the probability of occurrence is as high as $20 \%$. The main factor is that the local tissue of the oral cavity is degenerated or necrotic under the long-term action of orthodontic force. The mechanical force causes the protective substance on the surface of the root to fall off and the cementum is exposed. It is then absorbed by cementum cells ${ }^{[9]}$. Diphosphates have a P-C-P structure and are potent inhibitors of bone resorption, which are commonly used in the treatment of bone metabolismrelated diseases ${ }^{[10]}$. The mechanisms of bone resorption and alveolar bone resorption are generally the same, so whether bisphosphonates can be used for alveolar bone resorption caused by orthodontic treatment is a hot research topic.

Both periodontal disease and orthodontic treatment can cause bone resorption of the root bone or alveolar bone wrapped by the gingival gingival cells. In this study, clinical research, tissue sectioning, hematoxylin and eosin staining (HE staining) and other research methods were used to analyze the gingival bone resorption of rats in periodontal disease model, and the bisphosphate was used to analyze the bone absorption in tooth movement model rats.

\section{MATERIALS AND METHODS}

\section{Establishment of rat periodontal disease model:}

80 healthy clean grade Sprague-Dawley female rats weighing $200 \mathrm{~g}$ were selected and grouped, with 40 in the healthy controls group and 40 in the periodontitis experimental group. Rats were anesthetized by intraperitoneal injection of $3 \%$ sodium pentobarbital (40 mg/kg, China National Pharmaceutical Group Corporation, Shanghai Chemical Co., Ltd.), and then the neck of the left maxillary first molar was ligated using a No. 0.2 ligation wire. On the second day, intramuscular injection of predniso lone acetate
(Zhejiang Xianyu Pharmaceutical Co., Ltd.) was injected into the quadriceps of rats for three consecutive days, once a day, and each dose of $1.25 \mathrm{mg} / \mathrm{Kg}$. On the fifth day, the rats were anesthetized by intraperitoneal injection of $3 \%$ sodium pentobarbital, and the prepared pathogen mixture was inoculated orally (Porphyromonas gingivalis: Prevotella intermedia: Fusobacterium nucleatum=2:1:1, Procter \& Gamble), continuous inoculation for three days, once a day, each time $100 \mu \mathrm{l}$. Rats were fed until the fifteenth day, then anesthetized by intraperitoneal injection of $3 \%$ sodium pentobarbital. The rats were fixed and the ligation wire in the oral cavity was reviewed to ensure that the ligation wire entered the periodontal pocket as much as possible. The rats were kept feeding until the fifth week, and the modeling was completed.

\section{Establishment of rat tooth movement model:}

32 healthy and clean Sprague-Dawley female rats of about $200 \mathrm{~g}$ were selected, and rats were injected with $3 \%$ sodium pentobarbital $(40 \mathrm{mg} / \mathrm{kg}$ ) by intraperitoneal injection, and then anesthetized. The rat's head and limbs were then fixed so that it was lying on the operating table. Next, the appliance was installed on both sides of the maxillary dentition of the rat, in which the left side was the experimental group and the right side was the control group. Ibandronate sodium (Shenzhen Haiwang Pharmaceutical Co., Ltd.) is the latest generation of bisphosphonate, which has the advantages of low toxicity and stronger inhibition of bone resorption. $50 \mu \mathrm{l}$ of ibandronate $(500 \mu \mathrm{m} / \mathrm{l})$ was locally injected into the left first molar of the rat (experimental group) near the medial temporal mucosa three days before the installation of the appliance. On the right side (control group), $50 \mu$ of normal saline was injected in the same manner. The experimental group and the control group were injected every $3 \mathrm{~d}$ until the end of the subject.

The appliance installation can be divided into three steps. Firstly, the installation of the anterior device: firstly, the articaine adrenal gland $(1.7 \mathrm{ml}$ per branch, Acteon, French) was locally injected into the two central incisors located at maxilla. Then, using a diamond drill of a slow dental drill (SAESHIN, Korea), a small groove with a depth of $0.2 \mathrm{~mm}$ and a width of $0.5 \mathrm{~mm}$ was polished on the lingual surface, the distal middle surface, and the lip surface of the upper central incisors. Note that the small groove is next to the tooth neck and at the same level as the middle tangent. The entire grinding process was carried out under conditions of $0.9 \%$ saline cooling. Then, along the ground small groove, the two central incisors were 
ligated with orthodontic ligatures. The two middle incisors were wiped with alcohol cotton and dried, then etched with an acid etchant and rinsed with sterile water. In order to prevent the ligation wire from falling off and enhance the dental support ability, the two central incisors were bonded and fixed together with enamel adhesive (Hangzhou West Lake Biomaterial Co., Ltd.). Secondly, installation of the posterior device: a $0.2 \mathrm{~mm}$ ligature wire was passed through the gap between the first molar and the second molar and ligated in the middle of the first molar. Thirdly, the connection device: the orthodontic nickel-titanium spiral tension spring (0.012 inch in diameter, Yanyijin New Material Co., Ltd.) was used to connect the ligation wire of the first molar near the middle and the central incisor distally, a orthodontic force gauge was used to control the pull force to around $60 \mathrm{~g}$. After the orthodontic device was installed, the rats were intramuscularly injected with gentamicin $(5 \mathrm{mg} / \mathrm{Kg}$, Shandong Lukang Chenxin Pharmaceutical Co., Ltd.) for 3 consecutive d, once a day. Pay attention to the vital signs of the rats after the installation of the instrument, and keep warm measures until the rats fully recover their basic activities. In addition, the condition of the rat's body and appliance should be checked daily. The appliance was maintained to prevent it from falling off or damaging. 10 rats were randomly sacrificed on the $3^{\text {rd }}, 7^{\text {th }}$ and $14^{\text {th }} \mathrm{d}$ after the installation of the appliance, 5 of which were used for tissue sectioning and 5 for electron microscopy. 5 extra tooth movement model rats were prepared to prevent insufficient sample size caused by death in the rat during modeling.

\section{Specimen preparation:}

At the $3^{\text {rd }}, 7^{\text {th }}$ and $14^{\text {th }} \mathrm{d}$ of the experiment, 5 rats were randomly selected from each group. The rats were also anesthetized by intraperitoneal injection of $3 \%$ sodium pentobarbital $(40 \mathrm{mg} / \mathrm{kg})$, and the hair of the abdomen of the rats was cut off. Cut the chest and cut the ribs to expose the rat's heart. The perfusion needle was inserted into the aorta from the left ventricle. To prevent the perfusion needle from loosening, it is necessary to tie the wire tightly and fix it with a needle holder. The right atrium was then cut to expel blood to form a system of left ventricular perfusion in the right atrium. Initially, $200 \mathrm{ml}$ of normal saline was used for perfusion, and all of the blood in the rats was discharged; then, $40 \mathrm{ml}$ of $4 \%$ paraformaldehyde was slowly perfused with 1 drop per second, followed by infusion of $250 \mathrm{ml}$ of $0.9 \%$ paraformaldehyde at the same rate. When convulsions occur in the limbs of rats, it is indicated that paraformaldehyde has entered the brain. After the whole body tissue of the rat was hardened, the rat maxilla was peeled off, and the maxillary first molar and the surrounding maxillary tissue of the periodontal tissue were taken out and fixed in a $4 \%$ paraformaldehyde solution for $24 \mathrm{~h}$, then rinsed with distilled water for $60 \mathrm{~min}$, then $10 \%$ ethylenediaminetetraacetic acid (EDTA) decalcifying agent was added to completely soak the tissue for $10 \mathrm{w}$, pay attention to replace the decalcifying agent every $2 \mathrm{~d}$, and shake well during decalcification.

When the probe can be gently inserted into the specimen tissue, it indicates that the decalcification is complete. The tissue was raised with long flowing water. Next dehydration was carried out with $50 \%$, $75 \%$ and $100 \%$ ethanol respectively, and each gradient was used for dehydration twice; the xylene was added to soak the tissue to make the tissue transparent, and finally embedded in paraffin, and the preparation of the specimen was completed. The pathological slicer (LEICA, Germany) was used to slice 10 specimens along the long axis of the tooth near the root canal, and the thickness was $5 \mu \mathrm{m}$; the sections were fixed in polylysine (Wuhan Dr. Bioengineering Co., Ltd. Company) treated slides. Five of the sections were used to calculate the alveolar bone resorption index, and the other five sections were subjected to HE staining to observe osteoclasts.

\section{Comparison of clinical indicators of periodontal disease:}

Clinical indicators of periodontal disease are usually measured in three aspects: GI (gingival index), PLI (plaque index), and PD (probing depth).

GI is commonly used to indicate gingival lesions. Examination method: Use the periodontal probe to gently probe the periodontal pocket or sulcus of the live rat for about $10 \mathrm{~s}$. The gingival index was judged by the visual inspection and probing (Table 1).

PLI is used to evaluate oral hygiene status and to measure the prevention and treatment of periodontal disease. Inspection method: apply the cotton ball with plaque stain ( $2 \%$ basic fuchsin) to the experimental teeth of rat for $1 \mathrm{~min}$, then rinse for $30 \mathrm{~s}$ with sterile water to observe the area and depth of purple red on the tooth surface. Table 2 was used to determine the plaque index.

PD refers to the distance from the gingival margin to the bottom of the periodontal pocket or the bottom 
TABLE 1: GINGIVA INDEX JUDGMENT TABLE

\begin{tabular}{lc}
\hline Diagnosis & Gl \\
\hline $\begin{array}{l}\text { Mingiva health } \\
\text { gingiva color, mild edema, no bleeding during probing }\end{array}$ & 1 \\
$\begin{array}{l}\text { Moderate inflammation of the gingiva, red gingiva, } \\
\text { edema, bleeding }\end{array}$ & 2 \\
$\begin{array}{l}\text { Serious inflammation of the gingiva, obvious redness } \\
\text { or ulceration of the gingiva, and automatic bleeding } \\
\text { tendency }\end{array}$ & 3 \\
\hline
\end{tabular}

TABLE 2: PLAQUE INDEX JUDGMENT TABLE

\begin{tabular}{lc}
\hline Diagnosis & PLI \\
\hline Non plaque around gingival margin & 0 \\
$\begin{array}{l}\text { A thin plaque on the tooth surface near the gingival } \\
\text { margin }\end{array}$ & 1 \\
$\begin{array}{l}\text { Moderate plaque on the gingival margin or adjacent } \\
\text { surface }\end{array}$ & 2 \\
$\begin{array}{l}\text { A large number of plaques in the gingival sulcus and/ } \\
\text { or the gingival margin and adjacent occlusalf surface }\end{array}$ & 3 \\
\hline
\end{tabular}

of the sulcus. The clinical diagnose of the severity of periodontal disease is recorded in $\mathrm{mm}$. The depth of the gingival prosthesis of healthy gingiva is no more than $2 \sim 3 \mathrm{~mm}$. Under healthy conditions, the probe can enter the junctional epithelium; when there is inflammation, the probe will exceed the junctional epithelium, and enter the inflammatory zone to reach the crown of healthy connective tissue. After treatment, the infiltration of inflammatory cells in the connective tissue disappeared, the collagen fibers regenerated, and the resistance of the connective tissue to the probing was enhanced. The probe no longer penetrates into the connective tissue, but stopped in the junctional epithelium. Examination method: the depth of the sulcus at the three sites of mesiobuccal, buccal and distobuccal in the rats after sacrifice was measured with a William scaled periodontal probe, and the mean values were taken.

\section{Alveolar bone resorption in a rat model of periodontal disease:}

Alveolar bone is the most active part of the periodontal tissue and is also the most active part of the systemic skeletal system. Alveolar bone resorption is an important pathological change of periodontitis. Under physiological conditions, the absorption and regeneration of alveolar bone are balanced, and the height of the alveolar bone remains unchanged. When bone resorption increases, or bone regeneration decreases or both coexist, bone loss occurs, reducing the alveolar bone height. Due to the absorption of the alveolar bone, the supporting tissue of the tooth is lost, the tooth is gradually loosened, and finally fell off or removed. The rat model of periodontal disease was sacrificed by the method of spine dislocation. The skull was removed, boiled for $5 \mathrm{~min}$ and the flesh was removed and bone was retained. The teeth of the mesiolingual, glossa and the distolinguoincisal of the rat were selected and the length of the alveolar bone crest to the enamel bone boundary was measured with a vernier caliper (Shanghai Measuring Tool \& Blade Factory) under a magnifying glass, and the average value was taken.

\section{Determination of tooth movement distance in rat tooth movement model:}

The tooth movement model rats were sacrificed on the $3^{\text {rd }}, 7^{\text {th }}$ and $14^{\text {th }}$ respectively. The soft tissue was removed and the maxilla was dissected, and the periodontal tissues of the maxillary first molar region and its surrounding tissues were taken. The distance between the distal contact point of the maxillary first molar and the proximal mesial of the second molar was measured using a vernier caliper (accuracy of $0.02 \mathrm{~mm}$ ). Note that this procedure must be measured by the same laboratory staff, and each specimen was measured 5 times and the average value was taken.

\section{HE staining:}

The tissue was sectioned according to the above procedure, and the slide was dewaxed twice with toluene for 10 min each time; dehydrated with $100 \%, 90 \%$, $80 \%$ gradient ethanol for $3 \mathrm{~min}$; rinsed with distilled water for 3 to $5 \mathrm{~min}$; hematoxylin stained for $10 \mathrm{~min}$, rinsed with distilled water for 3 min; color separation was performed with $1 \%$ hydrochloric acid, rinsed with anti-blue for $30 \mathrm{~min}$; eosin stain for $5 \mathrm{~min}$, rinsed with distilled water for $10 \mathrm{~min}$; dehydrated with $80 \%$ ethanol for $1 \mathrm{~min}, 90 \%$ ethanol for $1 \mathrm{~min}$; xylene was used to make the slice transparent, and finally sealed with a neutral gingiva and HE staining was completed.

\section{Statistical analysis:}

The experimental results were expressed as mean 4-standard deviation. The statistical software PASW Statistics 18 (former SPSS) was used to analyze the data of each group by group $\mathrm{T}$ test. $\mathrm{p}<0.05$ was considered to be significantly different.

\section{RESULTS AND DISCUSSION}

The periodontal pockets or sulcus of the rats were gently probed for $10 \mathrm{~s}$ using periodontal probes. The gingival index statistics of normal rats and periodontal 
rats are shown in Table 3. Standard value $=($ GI $\times$ number of corresponding level mice)/total number of mice. The GI $(0.6 \pm 0.83)$ of normal rats was significantly lower than that of periodontal disease rats $(1.8 \pm 0.34)$ by group $T$ test, $\mathrm{p}<0.05$, which was statistically significant.

The plaque on the rat's teeth was stained, and the statistics of the area and depth of the purple color on the tooth surface were recorded based on Table 2 . The results are shown in Table 4 . Standard value $=(\mathrm{PLI} \times$ number of corresponding levels of mice)/total number of mice. PLI (1.8 \pm 0.75$)$ in normal rats was significantly lower than that in periodontal rats $(2.6 \pm 1.3), \mathrm{p}<0.05$ using statistical analysis by group $\mathrm{T}$ test. There was statistical significane.

The depth of the sulcus at the mesiobuccal, buccal, and distobuccal of the rats after sacrifice was measured by William's periodontal probe. The mean value was taken for statistical analysis. As shown in Table 5, compared with normal rats, the PD of periodontal rats increased significantly, $\mathrm{p}<0.05$, which was statistically significant.

The length of rat alveolar bone crest to enamel bone boundary was measured, and the mean value was used. Statistical analysis was performed by group $\mathrm{T}$ test. As shown in Table 6, it was found that the alveolar bone resorption of normal rats was significantly lower than that of periodontal disease rats. $p<0.05$, the difference was statistically significant. This suggested that periodontal disease can indeed lead to increased alveolar bone resorption inside the rat's gingiva.

\begin{tabular}{lccc} 
TABLE 3: & GI & STATISTICAL & RESULTS \\
PERIODONTAL DISEASE RATS & OF \\
\hline GI rating & Normal rat & Periodontal disease rat \\
\hline 0 & 5 & 0 \\
1 & 4 & 3 \\
2 & 1 & 6 \\
3 & 0 & 1 \\
\hline
\end{tabular}

TABLE 4: PLI STATISTICAL RESULTS OF PERIODONTAL DISEASE RATS

\begin{tabular}{lcc}
\hline PLI rating & Normal rat & Periodontal disease rat \\
\hline 0 & 1 & 0 \\
1 & 4 & 1 \\
2 & 2 & 2 \\
3 & 2 & 7 \\
\hline
\end{tabular}

TABLE 5: PD STATISTICS OF PERIODONTAL DISEASE RATS

\begin{tabular}{lcc}
\hline Grouping & Amount & PD \\
\hline Normal rat & 10 & $0.38 \pm 0.18$ \\
Periodontal disease rat & 10 & $1.60 \pm 0.21^{\mathrm{a}}$ \\
\hline
\end{tabular}

Note ${ }^{a}: p<0.05$

Special Issue 8, 2020

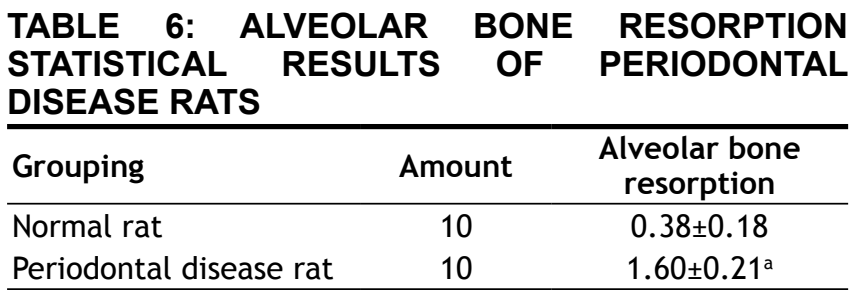

Note ${ }^{a}: p<0.05$

TABLE 7: RAT FIRST MOLAR MOVING DISTANCE
\begin{tabular}{lcc} 
(MM) & & \\
\hline Group & $\mathbf{3 ~ d}$ & $\mathbf{7 ~ d}$ \\
\hline Orthodontic treatment group & $0.13 \pm 0.04$ & $0.20 \pm 0.05$ \\
Orthodontic group & $0.14 \pm 0.07$ & $0.23 \pm 0.03$ \\
\hline
\end{tabular}

Note ${ }^{a}: p<0.05$

It can be observed from Table 7 that the movement distance of the first molar was also increased in the rat as time increased. There was no significant difference in the mean distance of tooth movement between the orthodontic treatment group and the orthodontic group between $0 \sim 3 \mathrm{~d}$ and $3 \sim 7 \mathrm{~d}(\mathrm{p}<0.05)$. In the $7 \sim 14 \mathrm{~d}$, compared with the orthodontic treatment group, the increase of the first molar movement distance in the orthodontic group began to increase significantly, indicating that ibandronate had a significant inhibitory effect on the bone resorption, with statistical significane $(p<0.05)$.

Cementoclast is eosinophilic cytoplasmic or multinucleated giant cells located in or near the absorption sulcus of the root surface. HE staining of periodontal tissue sections of rat model of periodontal disease showed that there was no pathological change in the junctional epithelium of the healthy group and periodontal disease group on day 3 (fig. 1). The gingival epithelium and sulcu epithelium were almost intact. The alveolar crest was not significantly absorbed and the alveolar bone was not damaged. The periodontal ligament fibers and the gingival fibers were arranged neatly, and a small amount of neutrophils infiltrated in the lamina propria. On the $7^{\text {th }} \mathrm{d}$ (fig. 2), no pathological change was observed in the healthy group, but the peritoneal fibers and gingival fibrous structures of the rat teeth in the periodontal disease group were loose, and the epithelium began to show signs of erosion. On the $14^{\text {th }} \mathrm{d}$ (fig. 3), no pathological changes were observed in the healthy group. In the periodontal disease group, the epithelial erosion was observed, the fiber arrangement was disordered and the fiber was broken, and inflammatory cells such as neutrophils were observed. Compared with the healthy group, there was no significant difference in the number of cementoclast in the periodontal disease group from 0 to $3 \mathrm{~d}(\mathrm{p}>0.05)$. 


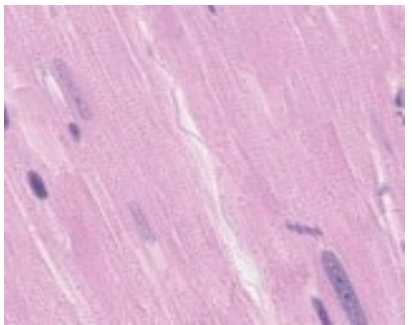

Healthy Rats

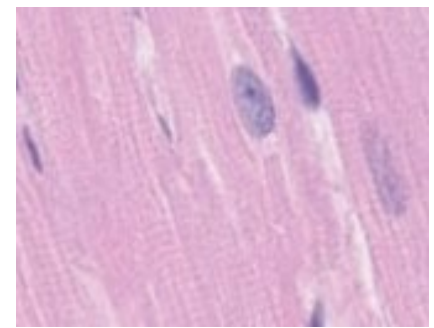

Periodontal Disease Group
Fig. 1: Periodontal tissue sections of healthy and periodontal rats by HE staining on day 3

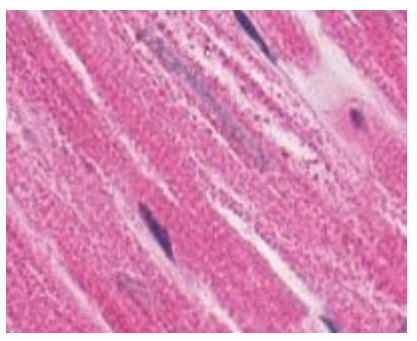

Healthy Rats

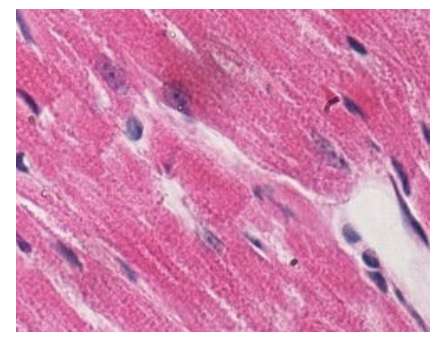

Periodontal Disease Group
Fig. 2: Periodontal tissue sections of healthy and periodontal rats by $\mathrm{HE}$ staining on day 7

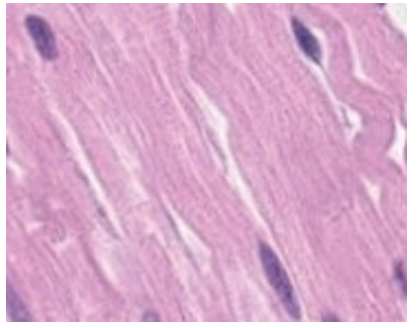

Healthy Rats

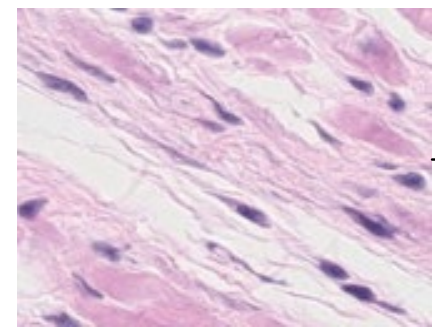

Periodontal Disease Group
Fig: 3: Periodontal tissue sections of healthy and periodontal rats by $\mathrm{HE}$ staining on day 14

During the 3-7 $\mathrm{d}$ and 7-14 $\mathrm{d}$, the number of cementoclast in the periodontal disease group increased significantly, with significant difference $(\mathrm{p}<0.05)$.

The periodontal tissue sections of the rat model of tooth movement were subjected to HE staining, and the number and morphology of the cementoclast were observed under a microscope (OLYMPUS, Japan) (fig. 4-6). In the period of $0 \sim 3 \mathrm{~d}$, the periodontal ligament of the first molar was narrower, and no obvious absorption lacuna was observed on the surface of the root and alveolar bone. In the process of $3 \sim 7$ and 7 14 d, absorption lacuna appeared in the root and alveolar bone. During the 7 14 d, the lacuna was obvious, and the nuclear giant cells accumulated a lot. The periodontal membrane fibers and the gingival fiber structure were loose, the fiber arrangement was disordered, and some fibers were broken. According to the statistical calculation of the number of cementoclast in the section, as shown in Table 8, there was no significant difference between the orthodontic group and the orthodontic treatment group at $0 \sim 3 \mathrm{~d}(\mathrm{p}>0.05)$; at 3 7 and 7 14 d, the number of cementoclast in the orthodontic treatment group was smaller than that in the orthodontic group, and the difference was statistically significant $(\mathrm{p}<0.05)$.

Using the image processing software Winceph 8.0, the root boundary and the absorption zone boundary of the tissue section were drawn, and the total area and absorption area of the root were calculated by computer, and the root absorption index=absorption area/total root area. As shown in Table 9, in the periodontal disease model, there was no significant difference in tooth root resorption between 0 and 3 days in the periodontal disease group ( $>0.05$ ), During the period

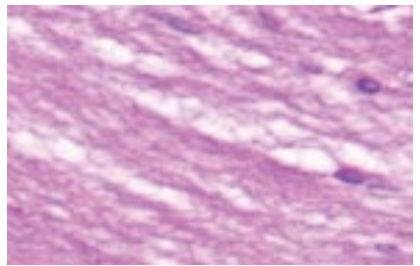

Orthodontic group

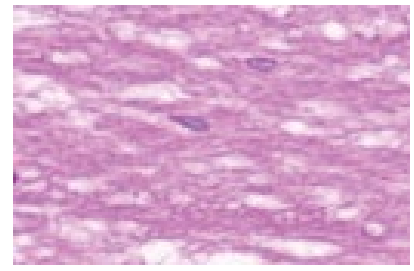

Orthodontic treatment group
Fig. 4: Periodontal tissue sections of orthodontic treatment and orthodontic treatment by HE staining on day 3

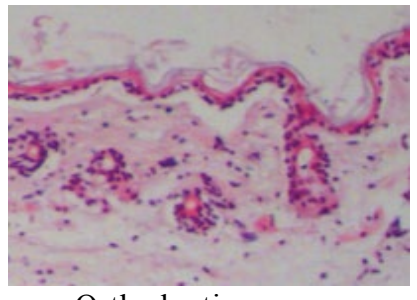

Orthodontic group

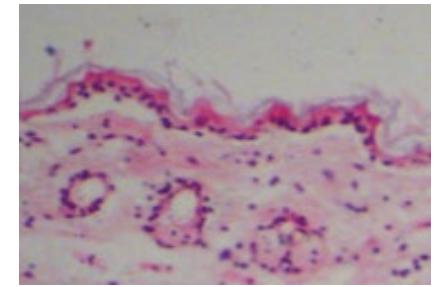

Orthodontic treatment group
Fig. 5: Periodontal tissue sections of orthodontic treatment and orthodontic treatment by HE staining on day 7

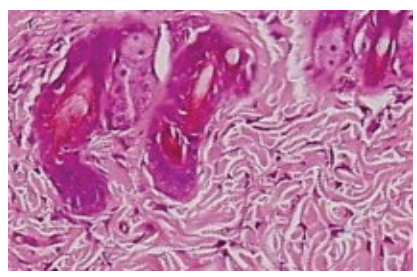

Orthodontic group

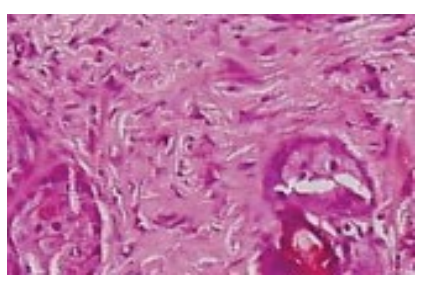

Orthodontic treatment group
Fig. 6: Periodontal tissue sections of orthodontic treatment and orthodontic treatment by HE staining on day 14

\section{TABLE 8: NUMBER OF CEMENTOCLAST}

\begin{tabular}{lcccc}
\hline Model type & Group & $3 \mathrm{~d}$ & $7 \mathrm{~d}$ & $14 \mathrm{~d}$ \\
\hline $\begin{array}{l}\text { Periodontal } \\
\text { disease model }\end{array}$ & Health group & 0.67 & $0.67^{\mathrm{a}}$ & $0.68^{\mathrm{a}}$ \\
& $\begin{array}{c}\text { Periodontal disease } \\
\text { group }\end{array}$ & 0.79 & $2.80^{\mathrm{a}}$ & $5.13^{\mathrm{a}}$ \\
$\begin{array}{l}\text { Tooth } \\
\text { movement }\end{array}$ & $\begin{array}{c}\text { Orthodontic group } \\
\text { Orthodontic }\end{array}$ & 0.77 & $2.73^{\mathrm{a}}$ & $4.91^{\mathrm{a}}$ \\
model & 0.76 & $2.54^{\mathrm{a}}$ & $3.78^{\mathrm{a}}$ \\
\hline
\end{tabular}

Note ${ }^{a}: p<0.05$ 
of 3 to $7 \mathrm{~d}$ and 7 to $14 \mathrm{~d}$, the periodontal absorption area of the periodontal disease group increased significantly, and the root resorption had significant difference $(p<0.05)$. In the tooth movement model, compared with the orthodontic group, there was no significant difference in the root resorption between 0 to $3 \mathrm{~d}$ in the orthodontic treatment group $(\mathrm{p}>0.05)$. During the period of 3 7 d and 7 14 d, the increase of periodontal area was significantly increased in the orthodontic treatment group, and there was significant difference in root resorption $(\mathrm{p}<0.05)$.

In this study, the clinical analysis of the periodontal disease model rats from the three aspects of gingival index, plaque index and depth of probing revealed that the periodontal disease group showed clinical pathological symptoms of periodontal disease compared with normal healthy rats. The pathological symptoms of the disease showed that the periodontal disease rats were successfully modeled. Subsequently, the first molar and its surrounding tissues of the periodontal disease rats were sectioned and observed under the microscope. Compared with the healthy group, the periodontal absorption area of the periodontal disease group increased significantly, that is, periodontal disease can indeed increase the degree of bone absorption in the alveolar bone. By counting the number of cementoclast in the tissue sections after HE staining, it was found that there were more cementoclast in the periodontal tissues of periodontal rats compared with healthy rats.

In the rat model of tooth movement, it was found that the tooth movement distance of rats treated with ibandronate was slower than that of the control group as the treatment time increased. Then, the first molar and its surrounding tissues of the tooth movement rat model were sectioned. It was found that the degree of bone resorption of the rats treated with topical application of Iban sodium phosphate was significantly reduced, and HE staining of the sections revealed that the number of cementoclast in the tissues was also significantly reduced.

In summary, it is found in periodontal disease model of rats that periodontal disease can promote the absorption of cementoclast of the lower teeth of the gingival bone, but whether it is related to the root exposure caused by periodontal disease still needs further analysis. In the rat tooth movement model, Iban sodium phosphate was found to have a significant inhibitory effect on root resorption induced by orthodontic treatment, but its mechanism and therapeutic effect on root resorption need to be further analyzed.

\section{REFERENCES}

1. Kesavalu L, Sathishkumar S, Bakthavatchalu V. Rat Model of Polymicrobial Infection, Immunity, and Alveolar Bone Resorption in Periodontal Disease. Infect Immun 2007;75(4):1704-12.

2. Nakane $S$, Kameyama Y. Root resorption caused by mechanical injury of the periodontal soft tissues in rats. J Periodontal Res 2010;22(5):390-5.

3. Grevstad H J. Doxycycline prevents root resorption and alveolar bone loss in rats after periodontal surgery. Scand J Dent Res 2010;101(5): 287-91.

4. Taubman M A, Valverde P, Han X. Immune response: the key to bone resorption in periodontal disease. J Periodontol 2005;76(11):2033-9.

5. Hausmann E. Potential Pathways for Bone Resorption in Human Periodontal Disease. J Periodontol 1974;45(5):338-43.

6. Reichart P A, Dürr U M, Triadan H. Periodontal disease in the domestic cat. A histopathologic study. J Periodontal Res 2010;19(1):67-75.

7. Liu L, Igarashi K, Haruyama N. Effects of local administration of clodronate on orthodontic tooth movement and root resorption in rats. Eur J Orthod 2004;26(5):469-73.

8. Goldie R S, King G J. Root resorption and tooth movement in orthodontically treated, calcium-deficient, and lactating rats. Am J Orthod 1984;85(5):424-30.

9. Cohen G, Campbell P M. Rossouw PEBuschang P H. Force magnitude and duration effects on amount of tooth movement and root resorption in the rat molar. Angle Orthod 2008;78(3):502-9.

10. Götz W, Kunert D, Zhang D. Insulin-like growth factor system components in the periodontium during tooth root resorption and early repair processes in the rat. Eur J Oral Sci 2010;114(4):318-27.

11. Haug S R, Brudvik P, Fristad I. Sympathectomy causes increased root resorption after orthodontic tooth movement in rats: immunohistochemical study. Cell Tissue Res 2003;313(2):167-75.

This is an open access article distributed under the terms of the Creative Commons Attribution-NonCommercial-ShareAlike 3.0 License, which allows others to remix, tweak, and build upon the work non-commercially, as long as the author is credited and the new creations are licensed under the identical terms

This article was originally published in a special issue, "Trends in Therapeutic Management of Various Clinical Conditions II" Indian J Pharm Sci 2021:83(2)Spl issue;81-87 